\title{
Directional Interpolation for Motion Weighted 4D Cone-Beam CT Reconstruction
}

\author{
Hua Zhang and Jan-Jakob Sonke* \\ Department of Radiation Oncology, The Netherlands Cancer Institute - Antoni van \\ Leeuwenhoek Hospital, Plesmanlaan 121, 1066 CX Amsterdam, The Netherlands \\ $\{$ h.zhang, j. sonke\}@nki.nl
}

\begin{abstract}
Image quality of four dimensional cone-beam computed tomography (4D CBCT) is limited by streaking artifacts due to insufficient projections after respiratory sorting. In this paper, a framework is proposed to combine improved motion and stationary regions of $\mathrm{CBCT}$ together to enhance the final reconstructed image. Firstly, streaking artifacts are decreased in the $4 \mathrm{D}$ CBCT by directional interpolation for additional cone-beam projections. Secondly, motion is estimated through deformable image registration of the $4 \mathrm{D}$ CBCT and motion proportional weights are assigned to each voxel. Finally, the weighted combination of the $3 \mathrm{D}$ and an interpolated $4 \mathrm{D}$ image is calculated. The proposed method is validated by both phantom and clinical data. Experiments demonstrate this method decreases streaking artifacts as well as image blur, and then improves image quality.
\end{abstract}

Keywords: 4D CBCT, directional interpolation, phase-correlated reconstruction.

\section{Introduction}

Cone-beam (CB) computed tomography (CT) is used to obtain patient images immediately before radiotherapy delivery. For patients with lesions in the thoracic and upper abdominal region, respiratory induced organ motion degrades reconstructed image quality. Phase-correlated four dimensional (4D) CBCT [1|2|3|4] was introduced to decrease respiratory induced blur and obtain time-resolved image information. These methods sorted acquired projections into nearly motionfree subsets according to a respiratory signal, and reconstructed each subset with FDK algorithm [5]. Since view-aliasing artifacts emerged due to insufficient number of projections after sorting, slowing gantry rotation [4] and multiple rotations [3] schemes were introduced to acquire more projections and improve image quality. McKinnon et al. [6] incorporated forward projection step to increase sampling projection number, while Bergner et. al. 7] introduced AAPC to distinguish the motion between respiratory and gantry-rotation on projection data, and applied a projectionwise phase-dependent weighting function. Then a phase-correlated

\footnotetext{
* This research was sponsored by CTMM, project Breast CARE (grant 03O-104).
} 
4D CBCT was reconstructed by combining high temporal resolution regions with strong motion and motionless regions with improved image quality. On the other hand, Betram et al. [8] employed directional interpolation to calculate intermediate views in 3D CBCT for skull imaging. Directional interpolation uses a structure tensor to estimate the local orientation of extracted sinograms, and interpolates pixels by applying the orientation. This method increases the number of projections and improves the reconstructed image quality. A complete comparison of different 4D methods is discussed in the paper [9].

In this paper, we introduce and evaluate a strategy called motion weighted 4D CBCT reconstruction. We firstly sort all projections into subsets according to respiratory signal after image acquisition. Directional interpolation is applied to each subset to increase projection number. All acquired original projections are reconstructed to a 3D $\mathrm{CBCT}$, and both interpolated and acquired projections are reconstructed to an interpolated 4D CBCT. Finally, according to the motion estimation results on this $4 \mathrm{D}$ data, we combine the $3 \mathrm{D}$ and $4 \mathrm{D}$ image by a weighting function. The proposed method is evaluated on both phantom and patient data.

\section{Methods}

The flow chart of our method is depicted in Fig 1. Directional interpolation is to reduce view aliasing artifacts and thus increase the image quality of $4 \mathrm{D}$ data. Motion estimation distinguishes moving and stationary anatomy in 4D CBCT. Finally, a new CBCT is generated based on the 3D CBCT reconstructed from all the original acquired projections for the stationary part, and based on the $4 \mathrm{D}$ interpolated reconstruction for the moving part.

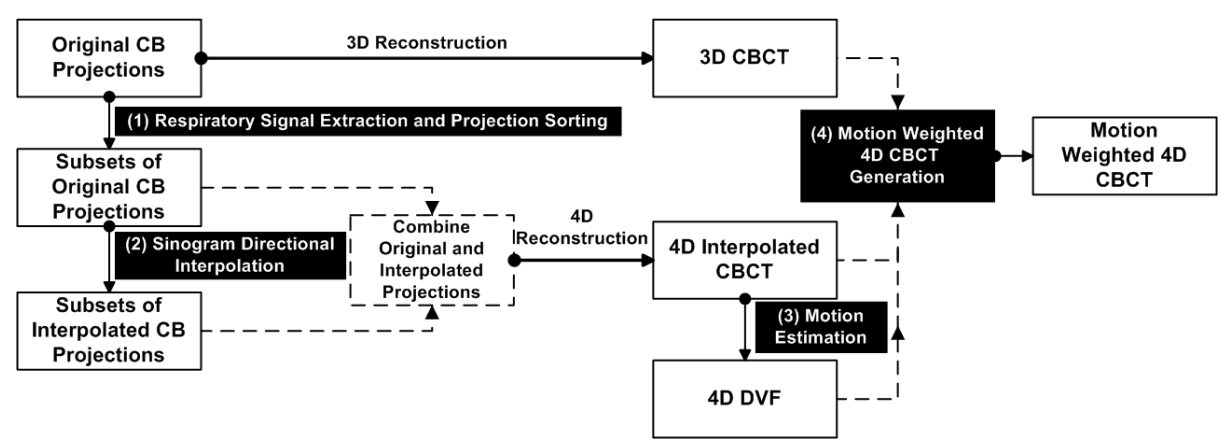

Fig. 1. Flow chart of our method. Rectangles are data and dashed rectangle means combining data from different source. The dark rectangles are main processing steps. FDK [5] is for 3D or 4D reconstruction (after sorting). 


\subsection{Respiratory Signal Extraction and Projection Sorting}

Phase-correlated 4D CBCT reconstruction requires a respiratory signal to sort projections into subsets according to the respiratory phase. This signal is extracted from the series of acquired original CB projections through image processing following the diaphragm motion [4. According to the phase of respiratory signal, we sort the projections into ten phase bins. In each bin, we collect a subset of projections which from the same respiratory phase. This process is illustrated in Fig 2. (a) where the bigger dots in phase graph represent the projections from the same phase bin.

\subsection{Sinogram Directional Interpolation}

Directional interpolation [8] is used to increase projection number. After projection sorting, all projections from the same phase bin are stacked together as a

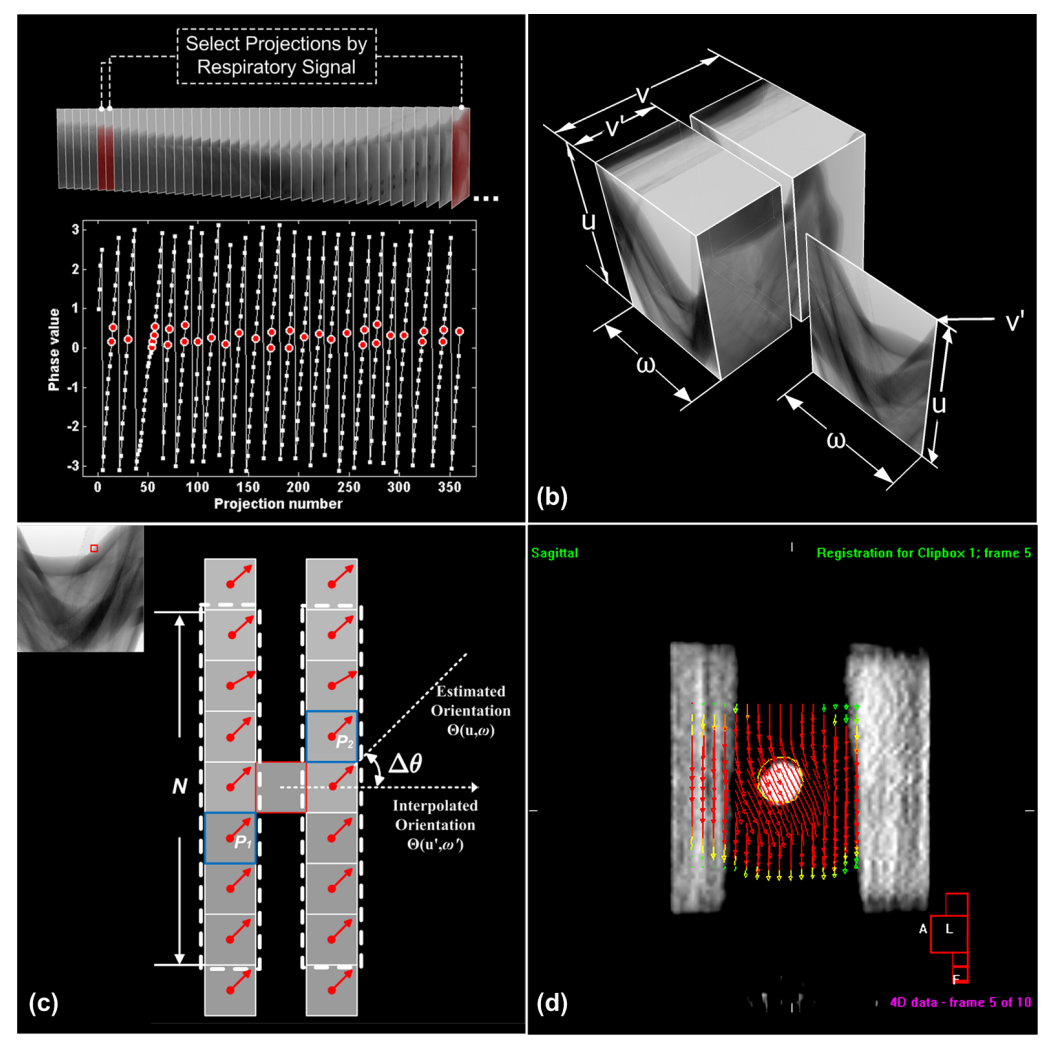

Fig. 2. (a) Selected cone-beam projections according to the respiratory signal. (b) The projections are stacked together and the sinogram is extracted. (c) The interpolated weight calculation. The dotted arrow represents the interpolated orientation $\theta_{\left(u^{\prime}, \omega^{\prime}\right) \rightarrow(u, \omega)}$, and the line arrows indicate the estimated orientations $\theta(u, \omega)$. (d) $4 \mathrm{D}$ deformation vector fields. 
cube $C(u, v, \omega)$ (Fig 2, (b)), where $u$ and $v$ represent the detector panel coordinate axes, and the axis $\omega$ is perpendicular to plane $(u, v)$ representing the gantry angle. Then a slice is extracted to form a sinogram. Since the respiratory motion is minimized by projection sorting, the projection pixel motion distance $|\boldsymbol{u}|$ is larger than $|\boldsymbol{v}|(|\boldsymbol{u}|$ is from gantry rotation and $|\boldsymbol{v}|$ is from respiratory motion). Therefore, the $2 \mathrm{D}$ sinogram $(u, \omega)$ is adequate to implement interpolation. The local orientation of the sinogram is defined as the directional axis which has the minimum pixel value variation in a local region. This direction is sought by calculating the eigenvectors of a structure tensor. Mixed-orientation-parameters [10] are used to detect multiple orientations, and a final single orientation is generated by setting threshold. A more detailed description on its implementation is introduced in paper [10].

Different weights $\{\eta(u, \omega),(u, \omega) \in \Omega\}$ are given to neighboring original pixels $(u, \omega) \in \Omega$ according to the orientation results. $\Omega$ is a region of all the neighboring pixels contributing to the interpolated pixel. Then the interpolated pixel value is calculated as

$$
i\left(u^{\prime}, \omega^{\prime}\right)=\frac{\sum_{(u, \omega) \in \Omega} \eta(u, \omega) \cdot i(u, \omega)}{\sum_{(u, \omega) \in \Omega} \eta(u, \omega)} .
$$

The weight $\eta$ is from $\eta(u, \omega)=\varepsilon \cdot[\cos (\Delta \theta)]^{4} \cdot \lambda$. Fig 2 (c) illustrates our interpolation weighting strategy. In this figure, $\left(u^{\prime}, \omega^{\prime}\right)$ is the interpolated location, $(u, \omega)$ is the neighboring pixel position, and $\Delta \theta=\theta(u, \omega)-\theta_{\left(u^{\prime}, \omega^{\prime}\right) \rightarrow(u, \omega)}$. The difference between estimated and interpolated orientation is quantified by $\Delta \theta$. When $\Delta \theta$ is closer to 0 , the interpolated pixel is closer to the local orientation, and higher weight is given to this neighbor pixel. We use $\cos (\Delta \theta)$ to smooth the weighting distribution in a none-linear shape. An indicator $\varepsilon$ is introduced as

$$
\varepsilon(x)= \begin{cases}1 & \text { if } \cos (\triangle \theta) \geq 0 \\ 0 & \text { otherwise }\end{cases}
$$

to eliminate these neighbor pixels where $\cos (\Delta \theta)$ is negative. This means the local orientation and interpolation direction are quite different. $\lambda=\left(1-e_{1} / e_{2}\right)$ is a ratio to specify local single orientation accuracy, and it means that the orientation estimation is more accurate when $\lambda$ is closer to $1 . e_{1}$ and $e_{2}$ are two eigenvalues from local orientation estimation [10] and $e_{1}<e_{2}$.

The interpolation function (11) also strongly depends on the interpolation neighborhood $\Omega$. The size of $\Omega$ is defined by $d=\left(s_{1} \cdot \Psi\right) /(m \cdot 2 \pi)$, where $s_{1}$ is the object motion distance along direction $u$ (Fig.2.(b)), $\Psi$ is total acquisition rotation angle, and $m$ is total acquired projection number for interpolation. During a full rotation acquisition, it is assumed that $\Psi=2 \pi, s_{1}$ equals to detector size. Then the average motion distance between adjacent projections is $s_{1} / \mathrm{m}$. In Fig.2.(c), the neighborhood size $N=d / 0.16$ pixels, and $0.16 \mathrm{~cm}$ is detector pixel size. 


\subsection{Motion Estimation}

The motion estimation algorithm analyzes the deformation between each phase of a $4 \mathrm{D}$ CBCT. The peak-inhale phase $3 \mathrm{D}$ CBCT is chosen as the reference, and other phases are registered to the reference. For the phantom data, local rigid registration by a shaped region of interest around a moving insert is used. For clinical data, an optical flow motion estimation method [11] was applied to calculate deformation vector fields (DVFs) between the reference image and other phase image. Then a $4 \mathrm{D}$ DVF is generated to express the respiratory motion of each voxel in a respiratory cycle.

\subsection{Motion Weighted 4D CBCT Reconstruction}

Our motion weighted reconstruction utilizes the 4D DVF to distinguish the moving and static part in the $4 \mathrm{D}$ CBCT, and combines these parts together according to the motion weight. From the 4D DVF, we calculate the displacement $\beta_{i}=\sqrt{v_{i 1}^{2}+v_{i 2}^{2}+v_{i 3}^{2}}$ for each voxel. $v_{i 1}, v_{i 2}, v_{i 3}$ are the motion in left-right (LR), cranial-caudal (CC) and anterior-posterior (AP) directions. In the 4D DVF, we find the largest displacement $\mathbf{B}_{j}=\max \left(\beta_{i}\right)$ in current phase $j$, and the motion weighted 4D CBCT is defined as $t(i, j)=\left(1-\omega_{i}\right) \cdot t_{s}(i)+\omega_{i} \cdot t^{\prime}(i, j)$, where $t_{s}$ is the $3 \mathrm{D} \mathrm{CBCT}, t^{\prime}$ is the interpolated CBCT, $j$ is current phase number, and $\omega_{i}=\beta_{i} / \mathbf{B}$.

\section{$3 \quad$ Experiments and Results}

\subsection{Data and Image Analysis}

In our experiments, the Dynamic Thorax Phantom (CIRC, Norfolk, USA) was scanned over an arc of $200^{\circ}$ with the acquisition times of 2,4 and 8 minutes. The phantom had a $3 \mathrm{~cm}$ diameter solid water sphere moving with $2 \mathrm{~cm}$ peak-topeak amplitude in the $\mathrm{CC}$ direction and 4 s period within lung density equivalent material. For the clinical study, a patient with respiratory tumor peak-to-peak amplitude of $1.1 \mathrm{~cm}$ in $\mathrm{CC}$ direction was scanned in 1 and 4 minutes. CB projections were acquired by a Synergy CBCT scanner (Elekta Oncology Systems, Crawley, UK). The flat imager has the specifications: $5.5 \mathrm{fps}, 40.96^{2} \mathrm{~cm}^{2}, 1024^{2}$ pixels; source-to-isocenter distance: $100 \mathrm{~cm}$, and source-to-panel distance 153.6 $\mathrm{cm}$. For computation efficiency, CB images were downsized to $256^{2}$ pixels $0.16^{2}$ $\mathrm{cm}^{2}$. CBCT volumes were reconstructed by FDK [5] with a resolution $0.2^{3} \mathrm{~cm}^{3}$ in a grid $128^{3}$. All voxel values were approximately normalized to Hounsfield Unit (HU).

For image analysis, a shaped region of interest (ROI) was manually defined from a 3D planning CT in phantom data, and the CBCT scan was subsequently registered to the planning CT. To assess streaking artifacts, root-meansquare-error (RMSE) between CBCT and planning CT was calculated as $E=$ $\sqrt{\frac{1}{n} \sum\left(t_{k}-t_{k}^{\prime}\right)^{2}}$, where $k \in \Omega_{1}, t_{k}$ was a voxel from reconstructed CBCT, $t_{k}^{\prime}$ 


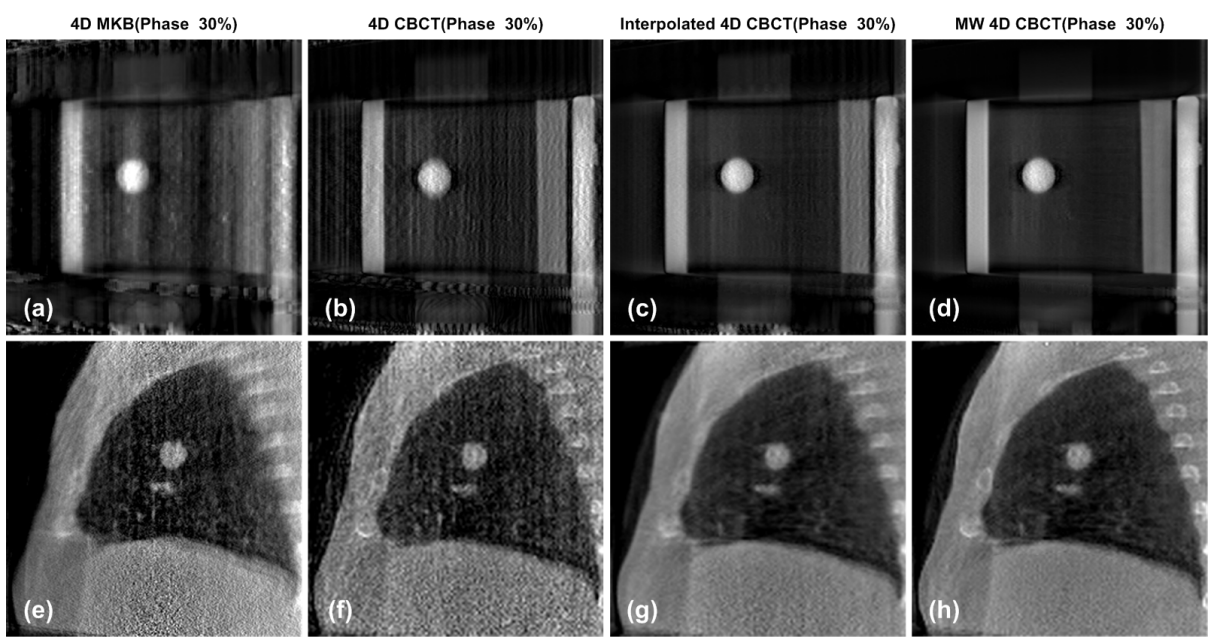

Fig. 3. Sagittal central slices of reconstructed images. (a)-(d) Phantom 4 min acquisition time. (e)-(h) Patient with $1.1 \mathrm{~cm}$ tumor motion, 4 min acquisition time. (a)-(h) Phase of $30 \%$ inhale-exhale cycle for the different time resolved reconstruction methods.

was a voxel from $\mathrm{CT}$ after it was resampled to the the grid of CBCT, and $n$ was the total voxel number inside $\Omega_{1} . \Omega_{1}$ was a manually segmented mask with setting threshold [1000, 1100] (HU) and erosion post-processing, and it comprised homogenous voxel values. The voxels from 3D CBCT (or each phase of $4 \mathrm{D} \mathrm{CBCT}$ ) were extracted in $\Omega_{1}$, and RMSE was calculated. Furthermore, image blur was defined in an extracted region $\Omega_{2}>1300(\mathrm{HU})$ of CT. Then $\Omega_{2}$ was mapped to the CBCT through image registration, and the average voxel value $\bar{t}=\frac{1}{k} \sum t_{k}, k \in \Omega_{2}$ was calculated. Region $\Omega_{2}$ contained high-contrast objects, and the decrement of $\bar{t}$ suggested the increment of image blur. For clinical study, the patient's gross tumor volume (GTV) was rigidly registered from CT to $\mathrm{CBCT}$ (or each $4 \mathrm{D} \mathrm{CBCT}$ phase). After registration, the correlation ratio 12] in GTV was used to quantify the image similarity of planning CT and reconstructed CBCT. FDK and MKB [6] were implemented to compare to our methods. 3D CBCT was reconstructed from full acquired projections by FDK. $4 \mathrm{D}$ CBCT and 4D interpolated CBCT were sorted into 10 respiratory bins. MKB was implemented by only one forward projection step and two backward projection steps.

\subsection{Results}

Fig 3 displays the sagittal slice of reconstructed $\mathrm{CBCT}$ by 4 minutes image acquisition time. 4D MKB (Fig 3 is blurred by forward interpolation.(a)(e)). Fig 3.(b)(f) belong to phase-correlated 4D CBCT and streaking artifacts are obvious, because only about $10 \%$ of the projections are used to reconstruct each phase. Interpolated 4D CBCT decreases streaking artifacts but induces image 

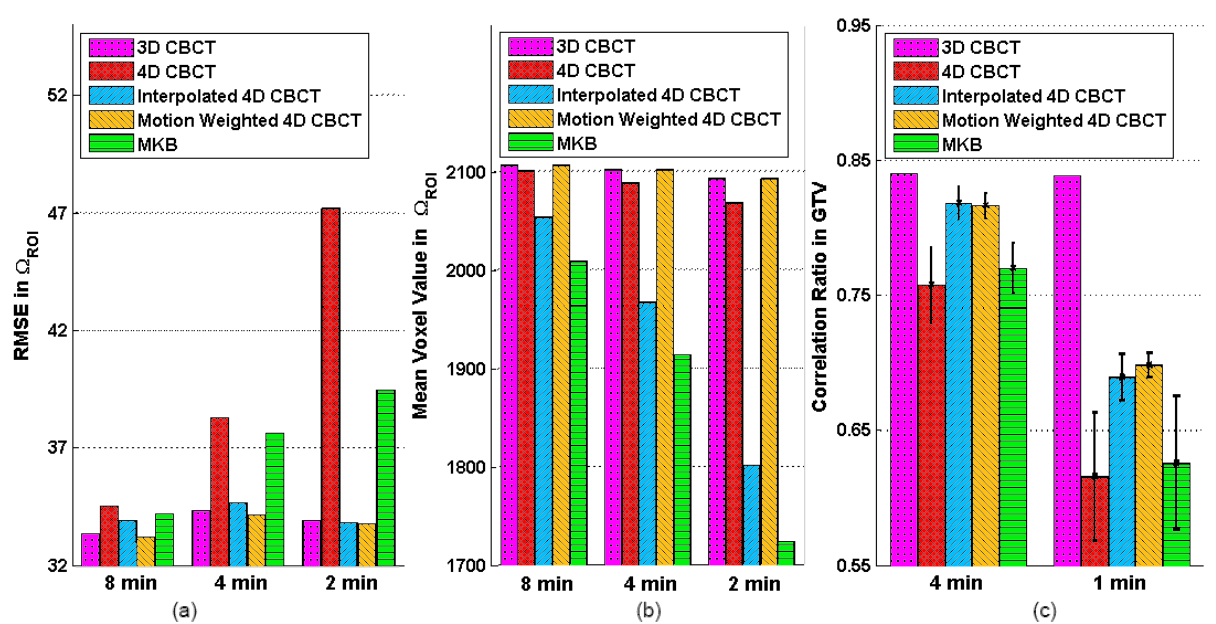

Fig. 4. (a) Streaking artifacts of phantom. (b) Image blur of phantom. (c) Correlation ratio of patient in GTV. Error bars are standard deviation calculated from 10 correlation ratios between $4 \mathrm{D}$ images and $\mathrm{CT}$.

blur. And finally motion weighted 4D CBCT preserves the sharpness of motionless bony structure and decreases the streaking artifacts.

The quantitative results are depicted in Fig 4 , and the results of $4 \mathrm{D}$ images are the mean value of all 10-phase images. In Fig.4.(a), streaking artifacts are compared between different acquisition protocols and methods. Interpolated and motion weighted 4D CBCT decrease the artifacts caused by undersampling of 4D CBCT. For highly undersampled 2 min scan, the streaking artifacts reduction is most pronounced. Image blur induced by interpolation is reduced by motion weighting strategy (Fig 4.(b)). Finally, Fig 4.(c) illustrates the correlation ratio of patient data. Since tumor motion is small, the correlation ratio between 3D $\mathrm{CBCT}$ and $\mathrm{CT}$ is close between different scanning times. The correlation ratio between interpolated and motion weighted CBCT is similar, but the decrease of standard deviations (error bars in Fig 4.(c)) suggests the improved image quality.

\section{Discussion and Conclusion}

In this paper, we have presented a motion weighted 4D CBCT reconstruction approach to cope with streaking artifacts and image blur. Streaking artifacts from undersampling were decreased by directional interpolation in sinogram space. MKB can be considered as an interpolation from all acquired projections, and our methods only utilize adjacent projections. Interpolation by more projections causes more image blur, and MKB has more blur than our method. Image blurring is a common phenomenon after interpolation, and depends on either the interpolation filter size or weights. Consequently, MKB and our method blur both interpolated projection and reconstructed images. The balance between 
image blur and streaking artifacts should be made according to the clinical purpose. Under the condition of sparse angle acquisition, our method boosts the interpolation filter to generate more discriminative weights, which is a effective strategy to decrease image blur and preserve the sharpness. Meanwhile, our method depends on 4D motion estimation accuracy. Our interpolation strategy decrease noise ratio and improves 4D motion accuracy. However, severe streaking artifacts can still possibly perturb 4D motion estimation and decrease the image quality of high-contrast objects such as bones and fiducial markers.

We have proposed a method with directional sinogram interpolation of respiratory correlated imaging, feeding into motion estimation in reconstruction space and motion estimation based weighting of 3D and interpolated 4D data sets. This method can decrease artifacts due to undersampling and constrains image blur after interpolation.

\section{References}

1. Dietrich, L., Jetter, S., Tücking, T., Nill, S., Oelfke, U.: Linac-integrated 4D cone beam CT: first experimental results. Physics in Medicine and Biology 51, 29392952 (2006)

2. Kriminski, S., Mitschke, M., Sorensen, S., Wink, N.M., Chow, P.E., Tenn, S., Solberg, T.D.: Respiratory correlated cone-beam computed tomography on an isocentric C-arm. Physics in Medicine and Biology 50, 5263-5280 (2005)

3. Li, T., Xing, L., Munro, P., McGuinness, C., Chao, M., Yang, Y., Loo, B., Koong, A.: Four-dimensional cone-beam computed tomography using an on-board imager. Medical Physics 33, 3825-3833 (2006)

4. Sonke, J.J., Zijp, L., Remeijer, P., van Herk, M.: Respiratory correlated cone beam CT. Medical Physics 32, 1176-1186 (2005)

5. Feldkamp, L.A., Davis, L.C., Kress, J.W.: Practical cone-beam algorithm. J. Opt. Soc. Am. A 1, 612-619 (1984)

6. Mckinnon, G.C., Bates, R.H.T.: Towards imaging the beating heart usefully with a conventional CT. IEEE Transactions on Biomedical Engineering BME-28(2), 123-127 (1981)

7. Bergner, F., Berkus, T., Oelhafen, M., Kunz, P., Pan, T., Kachelrieß, M.: Autoadaptive phase-correlated (AAPC) reconstruction for 4D CBCT. Medical Physics 36, 5695-5706 (2009)

8. Betram, M., Wiegert, J., Schäfer, D., Aach, T., Rose, G.: Directional view interpolation for compensation of sparse angular sampling in cone-beam CT. IEEE Transactions on Medical Imaging 28, 1011-1021 (2009)

9. Bergner, F., Berkus, T., Oelhafen, M., Kunz, P., Pan, T., Grimmer, R., Ritschl, L., Kachelrieß, M.: An investigation of 4D cone-beam CT algorithms for slowly rotating scanners. Medical Physics 37, 5044-5053 (2010)

10. Aach, T., Mota, C., Stuke, I., Mühlich, M., Barth, E.: Analysis of superimposed oriented patterns. IEEE Transactions on Image Processing 15, 3690-3700 (2006)

11. Wolthaus, J., Sonke, J.J., van Herk, M., Damen, E.: Reconstruction of a timeaveraged midposition CT scan for radiotherapy planning of lung cancer patients using deformable registration. Medical Physics 35, 3998-4011 (2008)

12. Roche, A., Malandain, G., Pennec, X., Ayache, N.: The Correlation Ratio as a New Similarity Measure for Multimodal Image Registration. In: Wells, W.M., Colchester, A.C.F., Delp, S.L. (eds.) MICCAI 1998. LNCS, vol. 1496, pp. 1115-1124. Springer, Heidelberg (1998) 